\title{
Evaporation of the planet HD 189733b observed in H I Lyman- $\alpha$
}

\author{
A. Lecavelier des Etangs ${ }^{1,2}$, D. Ehrenreich ${ }^{3}$, A. Vidal-Madjar ${ }^{1,2}$, G. E. Ballester ${ }^{4}$, J.-M. Désert ${ }^{1,2}$, \\ R. Ferlet ${ }^{1,2}$, G. Hébrard ${ }^{1,2}$, D. K. Sing ${ }^{1,2,5}$, K.-O. Tchakoumegni ${ }^{1,2,6}$, and S. Udry ${ }^{7}$ \\ 1 CNRS, UMR 7095, Institut d'astrophysique de Paris, 98bis boulevard Arago, 75014 Paris, France \\ e-mail: lecaveli@iap.fr \\ 2 UPMC Univ. Paris 6, UMR 7095, Institut d'Astrophysique de Paris, 98bis boulevard Arago, 75014 Paris, France \\ 3 Université Joseph Fourier-Grenoble 1/CNRS, Laboratoire d'astrophysique de Grenoble (LAOG) UMR 5571, BP 53, \\ 38041 Grenoble Cedex 09, France \\ ${ }^{4}$ Lunar and Planetary Laboratory, University of Arizona, 1541 E. University Blvd., Tucson, AZ 85721-0063, USA \\ 5 Astrophysics Group, School of Physics, University of Exeter, Stocker Road, Exeter EX4 4QL, UK \\ ${ }^{6}$ Lycée Passy Buzenval, 50 avenue Otis Mygatt, 92508 Rueil-Malmaison Cedex, France \\ 7 Observatoire de Genève, Université de Genève, 51 chemin des Maillettes, 1290 Sauverny, Switzerland
}

Received 24 September 2009 / Accepted 8 February 2010

\section{ABSTRACT}

\begin{abstract}
We observed three transits of the extrasolar planet HD 189733b in H I Lyman- $\alpha$ and in a few other lines in the ultraviolet with HST/ACS, in the search for atmospheric signatures. We detect a transit signature in the Lyman- $\alpha$ light curve with a transit depth of $5.05 \pm 0.75 \%$. This depth exceeds the occultation depth produced by the planetary disk alone at the $3.5 \sigma$ level (statistical). Other stellar emission lines are less bright, and, taken individually, they do not show the transit signature, while the whole spectra redward of the Lyman- $\alpha$ line has enough photons to show a transit signature consistent with the absorption by the planetary disk alone. The transit depth's upper limits in the emission lines are $11.1 \%$ for O I $\lambda 1305 \AA$ and $5.5 \%$ for C II $\lambda 1335 \AA$ lines.

The presence of an extended exosphere of atomic hydrogen around HD 189733b producing 5\% absorption of the full unresolved Lyman- $\alpha$ line flux shows that the planet is losing gas. The Lyman- $\alpha$ light curve is well-fitted by a numerical simulation of escaping hydrogen in which the planetary atoms are pushed by the stellar radiation pressure. We constrain the escape rate of atomic hydrogen to be between $10^{9}$ and $10^{11} \mathrm{~g} \mathrm{~s}^{-1}$ and the ionizing extreme UV flux between 2 and 40 times the solar value (1- $\sigma$ ), with higher escape rates corresponding to larger EUV flux. The best fit is obtained for $\mathrm{d} M / \mathrm{d} t=10^{10} \mathrm{~g} \mathrm{~s}^{-1}$ and an EUV flux $F_{\text {EUV }}=20$ times the solar value. HD 189733b is the second extrasolar planet for which atmospheric evaporation has been detected.
\end{abstract}

Key words. planetary systems - stars: individual: HD 189733

\section{Introduction}

\subsection{Evaporation of hot Jupiters}

Observations of the extrasolar planet HD 209458b in the Lyman- $\alpha$ line of atomic hydrogen (HI) have revealed that this planet is losing gas (Vidal-Madjar et al. 2003). Indeed, since the discovery of $51 \mathrm{Peg}$ b (Mayor \& Queloz 1995), the issue of the evaporation of hot Jupiters (also named "Pegasides", Guillot et al. 1996) has been raised by Burrows \& Lunine (1995) and Guillot et al. (1996). Nonetheless, the discovery of a large number of massive hot Jupiters ${ }^{1}$ and very hot Jupiters ${ }^{2}$ has led to the conclusion that the evaporation of massive planets has to be modest.

In this frame the discovery that the transiting extrasolar planet HD 209458b is indeed losing mass was rather unexpected. Lyman- $\alpha$ observations of the planetary transit showed excess absorption due to an extended $\mathrm{H}$ I cloud and provided a lower limit of the H I escape rate on the order of $10^{10} \mathrm{~g} \mathrm{~s}^{-1}$ (Vidal-Madjar et al. 2003; Vidal-Madjar \& Lecavelier des Etangs 2004). This discovery has been challenged by Ben-Jaffel (2007), but the apparent discrepancy has been resolved and the result obtained by Ben-Jaffel on this first data set strengthens the evaporation

1 Usually defined as massive planets orbiting in less than 10 days.

2 Usually defined as planets orbiting in less than 3 days (e.g., Gaudi et al. 2005). scenario (Vidal-Madjar et al. 2008). Two subsequent observations at low spectral resolution confirm the evaporation with the detection of a $5 \%$ absorption of the whole Lyman- $\alpha$ line, as measured using unresolved emission line flux during planetary transits observed with the Hubble Space Telescope (HST) STIS (Vidal-Madjar et al. 2004) and ACS instruments (Ehrenreich et al. 2010). An independent, new analysis of the low-resolution data set used by Vidal-Madjar et al. (2004) has confirmed that the transit depth in Lyman- $\alpha$ is significantly greater than the transit depth due to the planetary disk alone (Ben-Jaffel \& Sona Hosseini 2010). All of these three independent observations show a significant amount of gas at velocities exceeding the planet escape velocity, leading to the conclusion that HD 209458b is evaporating.

These observational constraints have been used to develop several theoretical models to explain and characterize the evaporation processes (Lammer et al. 2003; Lecavelier des Etangs et al. 2004, 2007, 2008a; Baraffe et al. 2004, 2005, 2006; Yelle 2004, 2006; Jaritz et al. 2005; Tian et al. 2005; García-Muñoz 2007; Holmström et al. 2008; Stone \& Proga 2009; Murray-Clay et al. 2009). Except for the model of Holmström et al. (2008) in which the observed hydrogen originates from charge exchange between a stellar wind of exotic properties and the planetary escaping exosphere (in this model the escape is found to be of the same order of magnitude), all of these modeling efforts have led to the conclusion that most of the EUV and X-ray 
Table 1. Log of the observations. Time is given in UT.

\begin{tabular}{llcccc}
\hline \hline \multirow{2}{*}{ Data set } & \multirow{2}{*}{ Date } & \multicolumn{2}{c}{ Observation } & \multicolumn{2}{c}{ Transit } \\
& & start & end & start & end \\
\hline Transit \#1 & $2007-06-10$ & $02: 04: 52$ & $07: 35: 03$ & $03: 36: 59$ & $05: 24: 28$ \\
Transit \#2 & $2007-06-18 / 19$ & $22: 43: 24$ & $04: 12: 45$ & $00: 35: 58$ & $02: 23: 27$ \\
Transit \#3 & $2008-04-24$ & $14: 01: 12$ & $17: 53: 22$ & $15: 00: 29$ & $16: 47: 58$ \\
\hline
\end{tabular}

energy input by the host star is used by the atmosphere to escape the planetary gravitational potential. Therefore, an "energy diagram" has been developed to describe the evaporation status of extrasolar planets, a diagram in which the potential energy of the planet is plotted versus the energy received by its upper atmosphere (Lecavelier des Etangs 2007). It has also been suggested that, in the case of planets with a mass of only a fraction of the mass of a hot Jupiter and orbiting close to their parent stars, evaporation can lead to significant modification of the nature of the planet, leading to the formation of planetary remnants (Lecavelier des Etangs et al. 2004). The recently discovered CoRoT-7b (Léger et al. 2009) could belong to a new category of planets, which was proposed for classification as "chthonian planets" (see Lecavelier des Etangs et al. 2004). A review of the evaporation of hot Jupiters can be found in Ehrenreich (2010).

However, up to now HD 209458b has remained the only planet for which an evaporation process has been observed. This raises the question of the evaporation state of other hot Jupiters and very hot Jupiters. Is the evaporation specific to HD 209458b or general to hot Jupiters? How does the escape rate depend on the planetary system and stellar characteristics? The discovery of HD 189733b, a planet transiting a bright and nearby K0 star $(V=7.7)$, offers the unprecedented opportunity to answer these questions. Indeed, among the stars harboring transiting planets, HD 189733 belongs to the brightest at Lyman- $\alpha$; therefore, despite the failure of the HST/STIS instrument one year before the discovery of HD 189733b, this planet remained one of the best targets when searching for atmospheric escape. In this paper we present transit observations of HD 189733b made with the UV channel of the Advanced Camera for Surveys onboard the Hubble Space Telescope (HST/ACS) using the same methodology as employed by Ehrenreich et al. (2010) for HD 209458b.

\section{2. $H D 189733 b$}

Located just 19.3 parsecs away with a semi-major axis of $0.03 \mathrm{AU}$ and an orbital period of 2.2 days, HD $189733 \mathrm{~b}$ belongs to the class of "very hot Jupiters" (Bouchy et al. 2005). More important, since this planet is seen to transit its parent star, the planetary transits and eclipses can be used to probe the planet's atmosphere and environment (e.g., Charbonneau et al. 2008; Désert et al. 2009).

HD 189733 b orbits a bright main-sequence star and shows a transit occultation depth of $\approx 2.4 \%$ at optical wavelengths (Pont et al. 2007). The planet has a mass $M_{\mathrm{p}}=1.13$ Jupiter masses $\left(M_{\mathrm{Jup}}\right)$ and a radius $R_{\mathrm{p}}=1.16$ Jupiter radii $\left(R_{\mathrm{Jup}}\right)$ in the visible (Bakos et al. 2006; Winn et al. 2007). The short period of the planet (2.21858 days) has been measured precisely (Hébrard \& Lecavelier des Etangs 2006; Knutson et al. 2009). Spectropolarimetry has measured the strength and topology of the stellar magnetic field, which reaches up to $40 \mathrm{G}$ (Moutou et al. 2007). Sodium has been detected in the planet's atmosphere by ground-based observations (Redfield et al. 2008). Using HST/ACS, Pont et al. (2008) detected atmospheric haze, which is interpreted as Mie scattering by small particles (Lecavelier des Etangs et al. 2008b). High signal-to-noise HST/NICMOS observations (Sing et al. 2009) have recently shown that the near-IR spectrum below $2 \mu \mathrm{m}$ is dominated by haze scattering rather than by water absorption (Swain et al. 2008). It has been tentatively proposed that CO molecules can explain the excess absorption seen at $4.5 \mu \mathrm{m}$ (Charbonneau et al. 2008; Désert et al. 2009). Using Spitzer spectroscopy of planetary eclipses, the infrared spectra of HD 189733b's atmosphere have revealed signatures of $\mathrm{H}_{2} \mathrm{O}$ absorption and possibly weather-like variations in the atmospheric conditions (Grillmair et al. 2008). A sensitive search with GMRT has provided very low upper limits on the meter-wavelength radio emission from the planet, indicating a weak planetary magnetic field (Lecavelier des Etangs et al. 2009).

\section{Observations}

Using the slitless prism spectroscopy mode of the Solar Blind Camera (SBC) of the HST/ACS, we observed 3 transits of HD 189733b. These observations provide 2D spectral images covering the far-UV wavelengths from $\sim 1200 \AA$ to $\sim 1700 \AA$. The log of the observations is given in Table 1 . We observed during four HST orbits for the first two transits, and during three HST orbits for the last transit. For each series of exposures within a given HST orbit, we obtained four individual exposures with the target at a first position on the detector, and four other exposures at a second position (dithering). We thus obtained a total of 32 exposures for transits \#1 and \#2 and 24 exposures for transit \#3. Each exposure had $257 \mathrm{~s}$ effective exposure time.

\section{Data analysis}

The data consist of 2D prism dispersed images of the star HD 189733. At these far-UV wavelengths, the spectrum of a $\mathrm{K}$-type star is composed of well-known bright chromospheric emission lines arising above a faint photospheric continuum. These emission lines form features that are seen as bright spots in the 2D images (Fig. 1). The brightest line is the H I Lyman- $\alpha$ at $\lambda 1216 \AA$ A. By identifying other features (e.g., H I, O I, C II, etc.) the spectrum can be wavelength-calibrated.

The aim of the data analysis is to obtain the best estimate of the brightness of each emission feature (with its associated error bar) in order to search for a possible transit signature. The first step is to subtract the background of each image. This background is caused mainly by the bright geocoronal Lyman- $\alpha$ airglow that produces a roughly uniform background entering the detector in the slitless prism mode. This background is accurately determined by the mean flux measured in each image above and below the stellar spectrum. We used two wide zones 100 pixels high centered 100 pixels above and below the spectrum. The background level is found to vary significantly within a given HST orbit. Nonetheless, various tests show that the background estimate is robust and its associated error is negligible in 


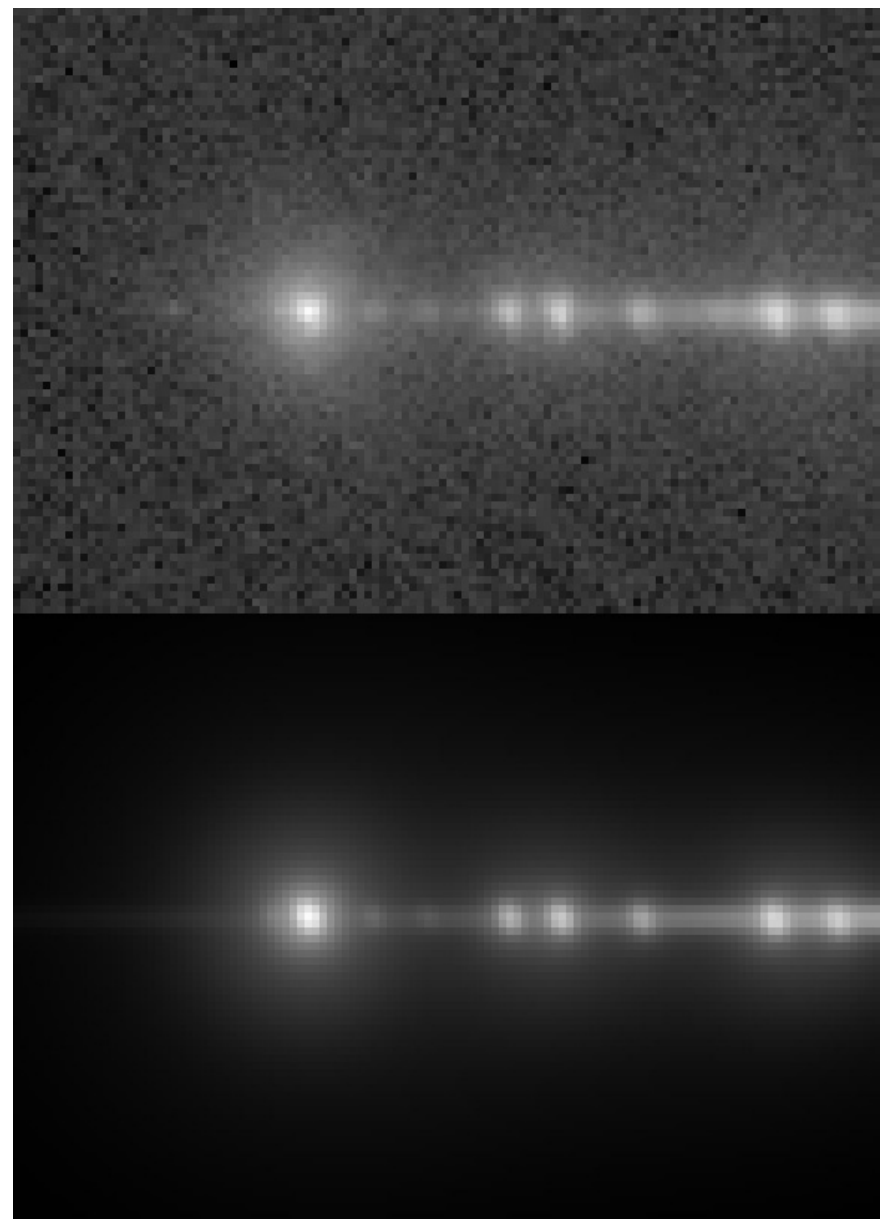

Fig. 1. 2D image of the HD 189733 spectrum obtained with ACS prism spectroscopy (upper panel). The bottom panel shows the 2D-model of this image. In this image, stellar emission lines can be seen as bright spots: from left to right, the six brightest spots are the Lyman-alpha line, followed by the two O I and C II emission features, further to the right is the SiIV feature, and the two last spots are emission by CIV and $\mathrm{CI}$. The stellar continuum emission is seen as a horizontal bright line along the spectrum.

the final error budget, well below the photon noise of all the measured emission features.

To estimate the brightness of each emission feature, we fitted each 2D spectral image with a parametric model and calculated the total number of counts in each line after subtracting the modeled 2D flux from the other emission features and from the continuum. For each 2D spectral image, the fit to the image was performed in 3 steps. First, we calculated a preliminary point spread function (PSF) by fitting the Lyman- $\alpha$ emission line. In the second step, the whole image was fitted with a model of six emission features whose positions were free to vary. In this fit, the initial guess for the features positions was set close to the six brightest spots as seen in Fig. 1 (H I, O I, C II, Si IV, C IV, C I). This second step was used to obtain a preliminary wavelength calibration by using the brightest emission lines. Finally, in a third step, we performed a fit of the whole 2D image in which the list of emission lines was extended to fainter lines. In this final stage the model included the wavelengths of the emission lines as a fixed parameter, while the wavelength calibration was free to vary and was defined by a 3rd order polynomial in the $X$ direction and a 1 st order polynomial in the $Y$ direction. The line list input to the model was defined by trial and error; few
Table 2. List of lines used to fit the 2D images.

\begin{tabular}{cl}
\hline \hline $\begin{array}{c}\text { Wavelength } \\
(\AA)\end{array}$ & Identification \\
\hline 1203.0 & N I + Si III \\
1215.7 & H I Ly- $\alpha$ \\
1240.8 & N v doublet \\
1264.7 & Si I I* \\
1305.4 & O I* + O I** \\
1335.1 & C II + C II* \\
1393.7 & Si IV \\
1402.8 & Si IV \\
1549.5 & C IV doublet \\
1656.9 & C I multiplet \\
\hline
\end{tabular}

misidentifications at the beginning of the process were easily corrected (even for faint lines) by checking the lines positions on a wavelength calibration plot. We ended up with a line list given in Table 2.

The intrinsic width of the observed emission lines is much smaller than the prism resolution, so the lines can be used to estimate the instrumental PSF. In all three steps of the fitting procedure, the PSF was modeled by the addition of six Gaussians. The sharpest Gaussian is an asymmetrical 2D Gaussian whose widths along the two axes and the orientation are free to vary. This sharp Gaussian has a width of about one pixel, and was found to vary from one exposure to the next; this is likely due to the telescope jitter. The five other Gaussians are axi-symmetrical Gaussians of fixed widths : $\sigma_{1}=2.5$ pixels, $\sigma_{2}=4$ pixels, $\sigma_{3}=6$ pixels, $\sigma_{4}=10$ pixels, and $\sigma_{5}=25$ pixels. These widths had been obtained by numerous experiments and allowed us to obtain the best fit to the data.

The stellar continuum was found to be well-fitted by an exponential law in the form $F_{\text {cont }}(\lambda)=a \exp (b \lambda)$, where $a$ and $b$ are free parameters. This stellar continuum model was also convolved with the PSF defined in the previous paragraph.

The resulting wavelength calibration (third degree polynomial) is plotted in Fig. 2 together with the features positions obtained at the second step of the fit. It is clear that the 3rd degree polynomial gives a satisfactory wavelength calibration. This calibration also allow correction for misidentifications. For instance, we first believed that the line between the $\mathrm{N}$ V doublet and the O I emission feature was a line of Si II from the ground level at $\lambda 1260.4 \AA$. But its measured position was significantly off the wavelength calibration. We thus realized that this line is in fact a line of Si II* at $\lambda 1264.7 \AA$, the $\mathrm{Si}$ II line being extincted by the interstellar medium absorption. To obtain the wavelength calibrated spectrum of HD 189733 plotted in Fig. 3,

we aligned all 2D images using the measured line positions, and we calculated the total spectrum by adding the measured counts along a band of 5 pixels height.

With the PSF parameters ( 8 parameters), the wavelength calibration (6 parameters), the brightness of each line (10 parameters for a total of 10 lines), the stellar continuum (2 parameters), and a free parameter to fit the residual background assumed to be constant within the image (1 parameter), each image is fitted with a total of 27 parameters.

Finally, after obtaining the best fit to each individual 2D image, we calculated a model image for each line using the best fit parameters, but changing the brightness parameter of the measured line to zero. This model image is subtracted from the data image, providing the best estimate of the line image in which the contribution of all other lines and of the continuum has been 


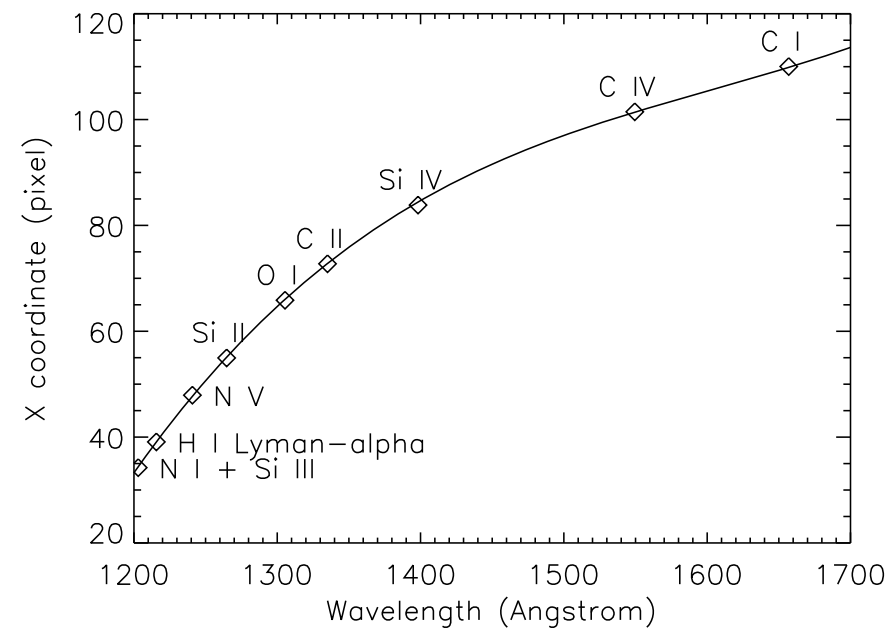

Fig. 2. Plot of the wavelength calibration. The squares show the position of each identified emission feature as a function of wavelength. The solid line shows the fit by a third-degree polynomial.

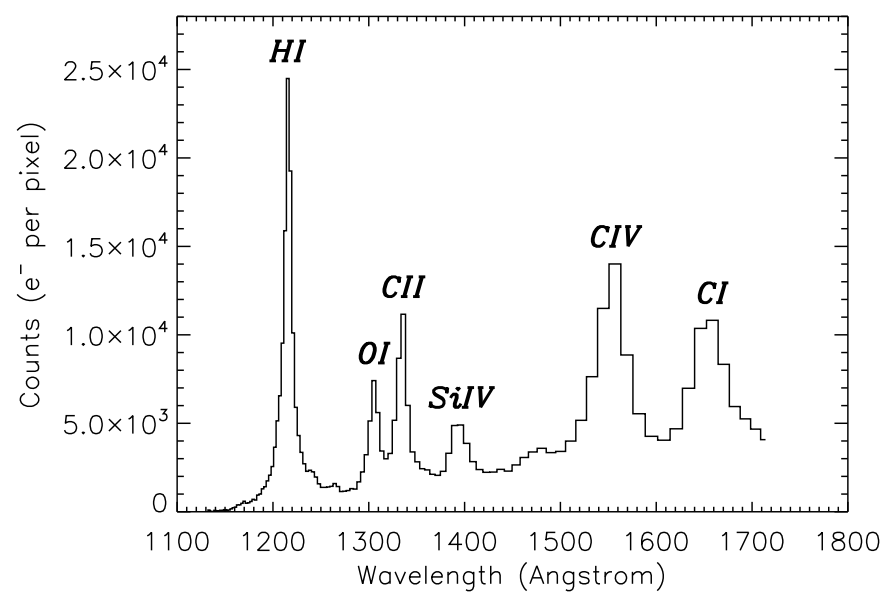

Fig. 3. Plot of one-dimensional wavelength calibrated ACS spectrum of HD 189733. The brightest lines are identified.

removed. We measured the brightness in the line image using aperture photometry. The error bars were calculated using the $2 \mathrm{D}$ image of the propagated errors provided by the instrument pipeline; these errors are mainly dominated by the photon noise. The resulting brightnesses were used to obtain the transit light curves plotted and discussed in Sect. 4.

\section{Transit light curves}

\subsection{HI Lyman- $\alpha$ light curve}

The main objective of these observations is to obtain the transit light curve in H I Lyman- $\alpha$ and to search for an atmospheric evaporation signature. For each of the three transits, we plotted the resulting light curves (Fig. 4). We fitted each light curve as a function of time by a transit model defined by a linear baseline for the stellar Lyman- $\alpha$ flux and the occultation by an opticallythick disk with the planetary ephemerides. The results from these three independent fits are given in Table 3.

We see that the second transit gives noisier measurements than the first one, and the observations were made only when the planet was off transit or during the partial occultation phase. In this transit \#2, no measurements during the total phase of the planetary transit were obtained because of the Earth occultation

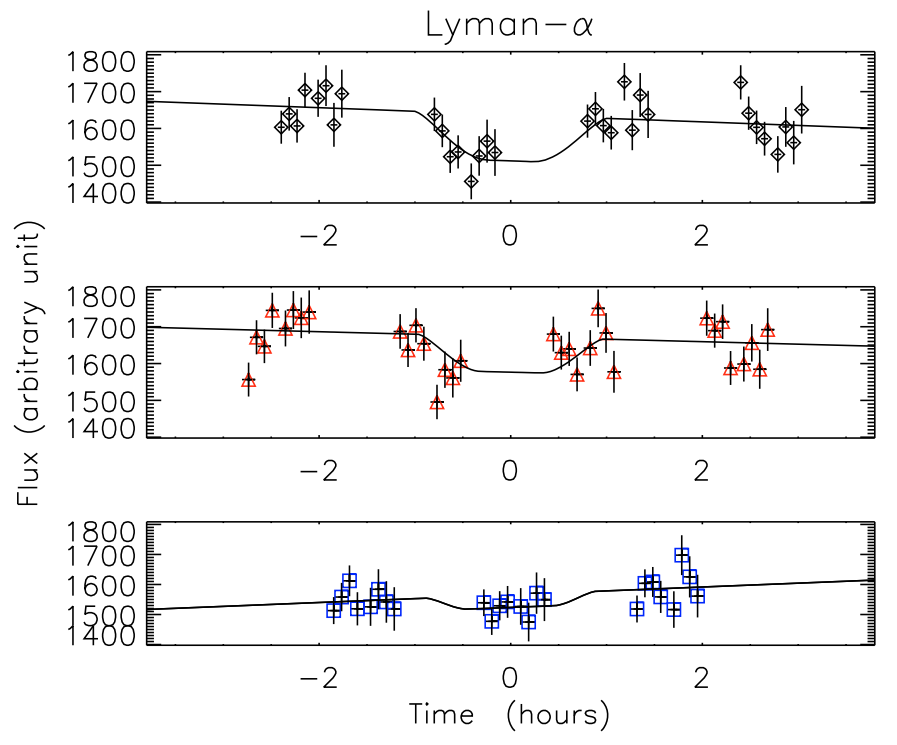

Fig. 4. Lyman- $\alpha$ flux as a function of time during three transits of HD 189733b. Different colors and symbols are for different epochs. The measurements in black diamonds, red triangles, and blue squares are for the 1st, 2nd, and 3rd transits, respectively. The curve shows the best fit to the data assuming a linear baseline and an optically-thick disk transiting the star. The transit depth (defined by the size of the occulting disk) is free to vary from one transit to another.

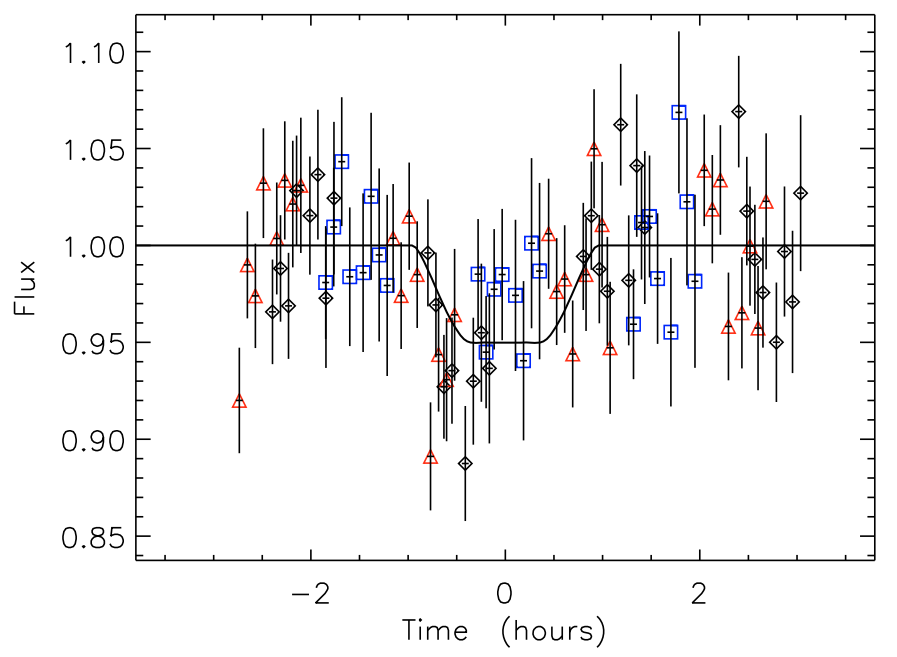

Fig. 5. Flux of the Lyman- $\alpha$ line as a function of time during transits of HD 189733b. The flux is normalized by the linear baseline as shown in Fig. 4 . The curve shows the best fit to the data by a single light curve obtained assuming the same transit depth for all transits. Symbols are the same as in Fig. 4.

of the target as seen by HST. In the first and third transits, the data covered all phases of the transit light curve, and measurements well before and after the transit allowed for estimates of the light curve baseline. Measurements obtained during the total phase of the planetary transit are well-fitted by a transit light curve of an optically-thick occulting disk (Fig. 4), providing an estimate of the size of the H I occulting cloud.

The full set of Lyman- $\alpha$ measurements can be phase-folded to provide a complete transit light curve (Figs. 5 and 6). The dispersion of the data rebinned by four is roughly divided by two, so there is no sign of red-noise in the data. The fit of this light curve yields a Lyman- $\alpha$ transit depth of $5.05 \pm 0.75 \%$. The error bars are estimated using the $\Delta \chi^{2}$ method (see e.g., 
Table 3. Results from the Lyman- $\alpha$ light curve.

\begin{tabular}{cccccc}
\hline \hline Data set & $\chi^{2}$ & $\begin{array}{c}\text { Degree of } \\
\text { freedom }\end{array}$ & $\begin{array}{c}\text { Absorption } \\
(\%)\end{array}$ & $\begin{array}{c}\sqrt{\Delta \chi^{2}} \\
\text { from 0\% abs. }\end{array}$ & $\begin{array}{c}\sqrt{\Delta \chi^{2}} \\
\text { from 2.4\% abs. }\end{array}$ \\
\hline Transit \#1 & 29.4 & 29 & $7.6 \pm 1.5$ & $5.7 \sigma$ & $3.8 \sigma$ \\
Transit \#2 & 48.8 & 29 & $5.6 \pm 1.7$ & $3.3 \sigma$ & $2.0 \sigma$ \\
Transit \#3 & 12.7 & 21 & $2.7 \pm 1.2$ & $2.2 \sigma$ & $0.2 \sigma$ \\
All & 97.9 & 81 & $5.05 \pm 0.75$ & $6.4 \sigma$ & $3.5 \sigma$ \\
\hline
\end{tabular}

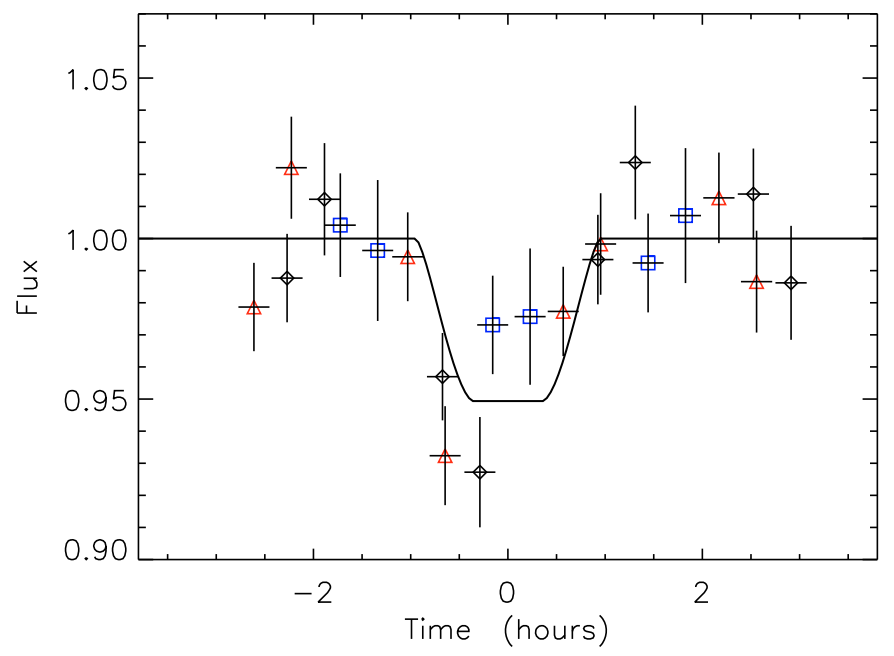

Fig. 6. Same as previous plot in which the data have been rebinned by four. The possible difference of transit depth between different epochs are visible here.

Hébrard et al. 2002), and using the same method we can estimate the significance of the detection by calculating the difference of $\chi^{2}$ between the best fit and a fit with no absorption at all (5th column of Table 3 ) or a fit with an occultation by the planetary disk alone (6th column of Table 3). Our result shows a transit depth that appears to be deeper than the occultation by the planetary disk alone $(\sim 2.4 \%)$. Using the statistical error bars, the excess absorption is at $3.5 \sigma$ level. Of course, an unknown systematic effect mimicking a transit light curve with an excess absorption cannot be excluded; nonetheless, the most likely explanation is the presence of a huge cloud of atomic hydrogen surrounding HD $189733 b$.

The transit depths measured on individual light curves of the transits \#1 and \#3 are different by about $2.5 \sigma$. This difference is clearly visible in the plot with data rebinned by four (Fig. 6).

\subsection{Light curves in other lines}

Using the same procedure as for Lyman- $\alpha$, we measured the brightness of other stellar emission lines and fit the light curves to measure the transit occultation depths (Table 4). In these light curves, few measurements are obviously outliers, in particular in the curve of the $\mathrm{CI}$ line. These outliers have been removed by flagging the points beyond $3 \sigma$ of the best fit. The number of points used for the fit is given in the third column of Table 4.

We detect a transit signature in none of these lines. Only upper limit on the transit depths can be derived. For each line, the $3-\sigma$ upper limit corresponds to the transit depths that yield a $\chi^{2}$ of 9 above the $\chi^{2}$ of the best fit to the data. For the two most important species (O I and C II), we obtain a 3- $\sigma$ upper limit for the transit depth of about $11 \%$ and $5 \%$, respectively (Fig. 7). These two species are abundant and light; therefore they should be, after hydrogen, the most abundant species in the upper atmosphere. In contrast to the case of HD 209458b (Vidal-Madjar et al. 2004), the non-detection of oxygen and carbon in the upper atmosphere of HD $189733 \mathrm{~b}$ precludes us from concluding anything about the nature of the escape mechanism (see also Ben-Jaffel \& Sona Hosseini 2010). Note, however, that the lines from the ground level of O I and C II are expected to be strongly absorbed by the interstellar medium (see Fig. 2 of Vidal-Madjar et al. 2004), so the observed stellar emission lines are mainly from the excited levels $\mathrm{OI}^{*}, \mathrm{OI}^{* *}$, and $\mathrm{CI} *$. Therefore, the upper limits quoted above should be interpreted with caution, keeping in mind that these levels are collisionally populated in gas with typical densities above $\sim 10^{6} \mathrm{~cm}^{-3}$.

\subsection{Total flux redward of Lyman- $\alpha$}

The planet transit cannot be seen in stellar emission lines other than Lyman- $\alpha$ (Sect. 4.2). This is caused by the very low number of photons available in those lines. For instance, there are about 200 photons per exposure in the O I feature at $1305 \AA$; with a dozen of exposures during the planetary transit, the accuracy of the transit depth is photon-noise limited to about $\sim 2 \%$. This limitation can be overcome by the adding photons in a wide zone of the spectra redward of the Lyman- $\alpha$ line, including all these other emission lines and the stellar continuum together. Using the range from $1280 \AA$ to $1700 \AA$, we find about 3000 photons per exposure (corresponding to about $0.5 \%$ photon-noise limit on the transit depth measurement), to be compared with the 1700 photons per exposure in the Lyman- $\alpha$ line. We calculated the corresponding light curve (Figs. 8 and 9) and found a clear transit signature. In this light curve, we also see a flarelike feature superimposed on the transit light curve ${ }^{3}$. This flare took place in the middle of the third visit, at the same epochs as outliers already seen in light curves of individual lines, strengthening the need for flagging those measurements. For the spectra redward of Lyman- $\alpha$, if outliers beyond 3- $\sigma$ are flagged, we find a transit depth of $2.7 \pm 0.6 \%$, which is consitent with the transit of the planet with a depth of $2.4 \%$ (Table 5). This $4.6 \sigma$-detection of the planet transit as expected in such data gives confidence to the detection of the transit seen at Lyman- $\alpha$. The calculated error bars on the depths of both these detected transits are also consistent with the theoretical limit from photon noise.

Finally, the detection of a flare in the middle of the third transit could explain why the transit depth in Lyman- $\alpha$ is shallower in this last observation. If the measurements obtained at the epoch of the flare are flagged (the epoch of the 3 outliers at 3- $\sigma$ plus the two adjacent points), we find a Lyman- $\alpha$ transit depth of $5.7 \pm 0.9 \%$. With this last result, we would reach exactly the same conclusions. Also, a careful inspection of individual measurements does not show any hint of flare in Lyman- $\alpha$

3 This flare-like feature looks similar to a flare seen at optical wavelength during the transit of the planet OGLE-TR-10b (Bentley et al. 2009). This topic will be discussed further in a separate paper. 
Table 4. Results from light curves of emission lines other than H I Lyman- $\alpha$.

\begin{tabular}{cccccccc}
\hline \hline Line & $\begin{array}{c}\lambda \\
(\AA)\end{array}$ & $\begin{array}{c}N \\
\text { measurements }\end{array}$ & $\chi^{2}$ & $\begin{array}{c}\text { Degree of } \\
\text { freedom }\end{array}$ & $\begin{array}{c}\text { Absorption } \\
(\%)\end{array}$ & $\begin{array}{c}\sqrt{\Delta \chi^{2}} \\
\text { from 2.4\% abs. }\end{array}$ & $\begin{array}{c}\text { 3- } \sigma \text { upper limit } \\
(\%)\end{array}$ \\
\hline OI & 1305 & 87 & 80.5 & 80 & $3.7 \pm 2.4$ & 0.5 & 11.1 \\
CII & 1335 & 88 & 80.7 & 81 & $1.0 \pm 1.0$ & 1.3 & 5.5 \\
SIV & 1400 & 86 & 79.6 & 79 & $1.6 \pm 1.6$ & 0.8 & 11.2 \\
CIV & 1550 & 86 & 94.1 & 79 & $1.7 \pm 1.7$ & 0.5 & 7.0 \\
CI & 1660 & 84 & 66.2 & 77 & $0.7 \pm 0.7$ & 1.5 & 6.2 \\
\hline
\end{tabular}

CII

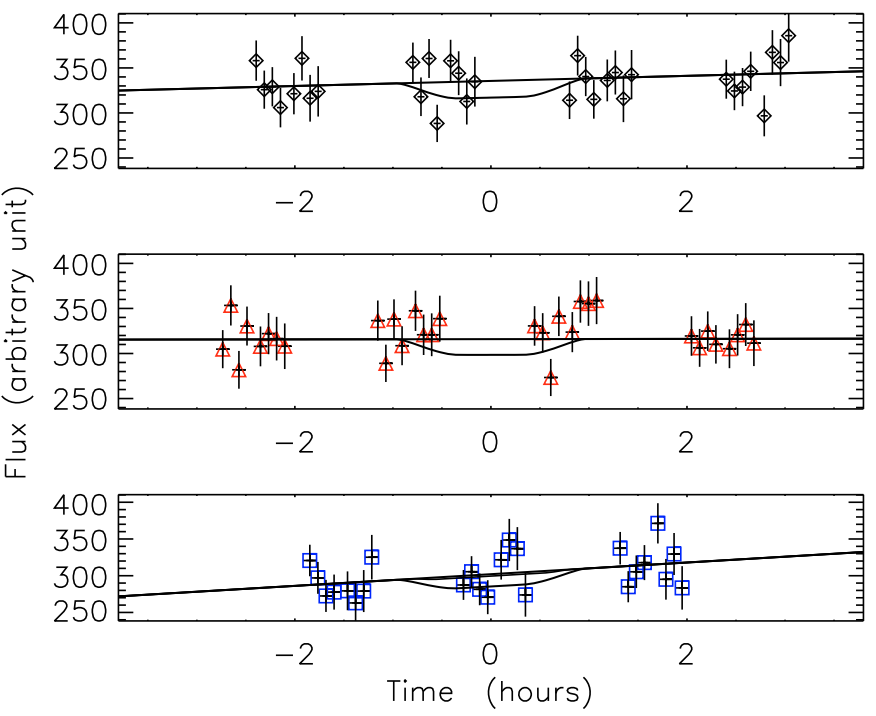

Fig. 7. Plot of the flux in the C II line for the three HST transits. The data are photon-noise limited and the transit of the planet is not detected. Only an upper limit on the transit depth can be obtained. The solid line shows the transit light curve with the 3- $\sigma$ upper limit on the transit depth.

(Fig. 4) or in the O I line, in contrast to carbon lines (C II plotted in Fig. 7 and C IV), which show suspiciously large flux at the epoch of the flare. This behavior is similar to what has been seen for solar flares, where large variations are seen in C IV lines, while the Lyman- $\alpha$ line that originates in a different part of the stellar atmosphere barely shows variations (e.g., Brekket et al. 1996). We conclude that the flare does not affect the main results of the present paper. The detection of the transit of the planet in the spectra redward of Lyman- $\alpha$ strengthens the results at Lyman- $\alpha$.

\section{Evaporation of HD 189733b}

\subsection{The stellar Lyman- $\alpha$ emission line profile}

As shown in Sect. 4.1, we detect a transit signature in the light curve of the Hi Lyman- $\alpha$ stellar emission line with a depth of $\sim 5 \%$, significantly deeper than the transit $2.4 \%$ depth due to the planetary disk alone, as seen at optical wavelengths. Moreover, the HD 189733b Lyman- $\alpha$ line is a broad emission line, with a width of $\sim \pm 150 \mathrm{~km} \mathrm{~s}^{-1}$. From the experience with HD 209458b, we know that to interpret this measurement we must consider the possibility that the hydrogen cloud surrounding HD $189733 \mathrm{~b}$ only absorbs a fraction of this stellar line width. Therefore the measured 5\% absorption depth in the total flux of the line is possibly caused by a larger absorption of only a fraction of the line width (see discussion in

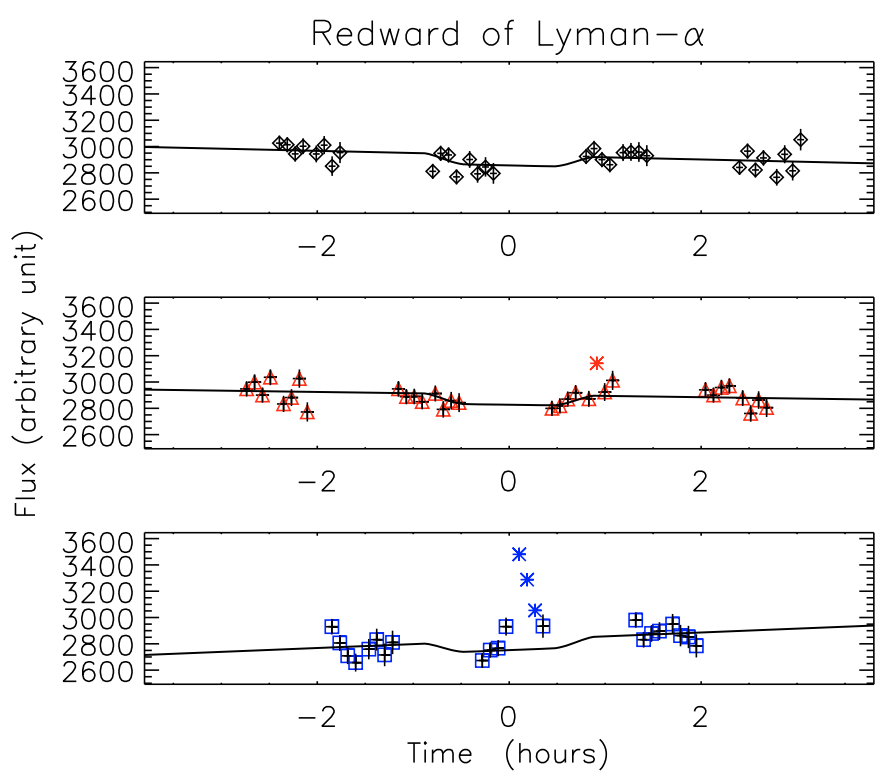

Fig. 8. Plot of the flux redward of Lyman- $\alpha$ for the three HST transits. Some measurements are clearly outliers; measurements beyond 3- $\sigma$ from the best fit are plotted with stars. Symbols are the same as in Fig. 4

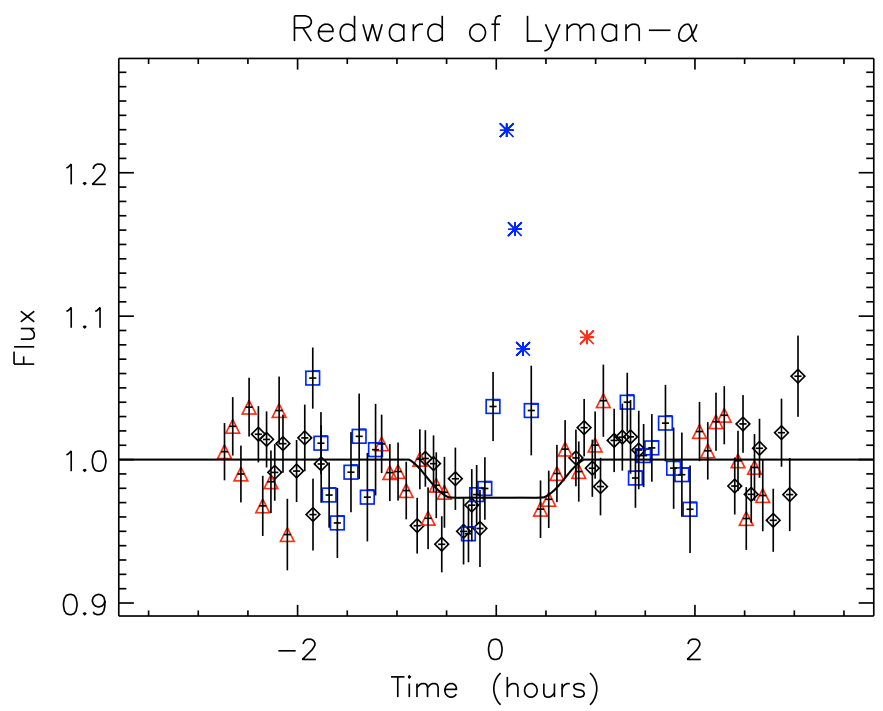

Fig. 9. Plot of the flux redward of Lyman- $\alpha$. The time has been phasefolded and the flux normalized by the linear baseline shown in Fig. 8. Outliers at 3- $\sigma$ are plotted with stars.

Vidal-Madjar et al. 2008). To interpret the measured absorption depth, it is therefore important to have an estimate of the emission line width and shape. 
Table 5. Results from the light curve redward of Lyman- $\alpha$.

\begin{tabular}{cccccccc}
\hline \hline Data & $\begin{array}{c}\lambda \\
(\AA)\end{array}$ & $\begin{array}{c}N \\
\text { measurements }\end{array}$ & $\chi^{2}$ & $\begin{array}{c}\text { Degree of } \\
\text { freedom }\end{array}$ & $\begin{array}{c}\text { Absorption } \\
(\%)\end{array}$ & $\begin{array}{c}\sqrt{\Delta \chi^{2}} \\
\text { from 0\% abs. }\end{array}$ & $\begin{array}{c}\sqrt{\Delta \chi^{2}} \\
\text { from 2.4\% abs. }\end{array}$ \\
\hline redward of Ly- $\alpha$ & $\geq 1280$ & 84 & 96.5 & 77 & $2.7 \pm 0.6$ & $4.6 \sigma$ & $0.4 \sigma$ \\
\hline
\end{tabular}

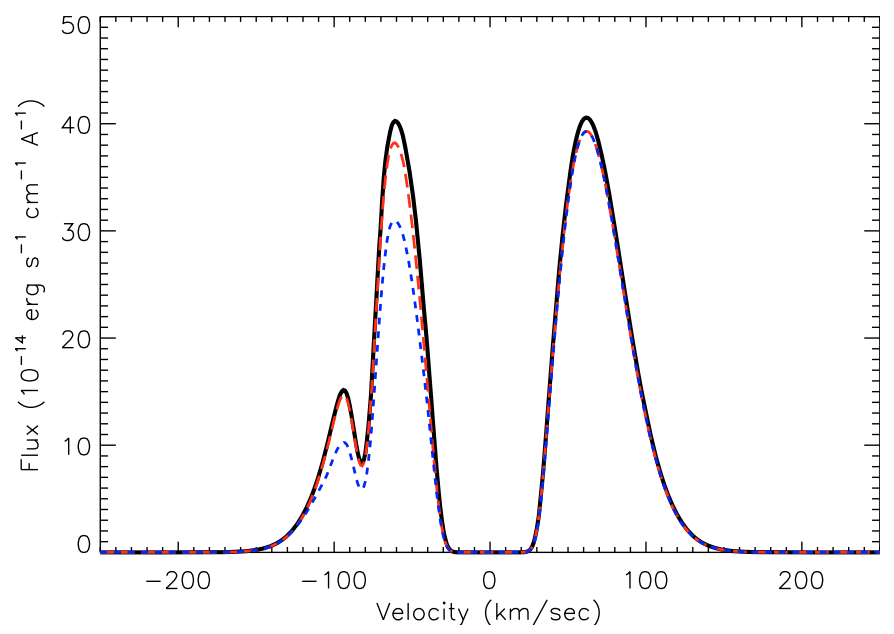

Fig. 10. Plot of the theoretical spectrum of the Lyman- $\alpha$ line of HD 189733. The line shape takes the absorption by the interstellar medium hydrogen and deuterium into account (black thick line). Two different spectra, including absorption by the planetary exophere when it passes in front of the star, are superimposed (dashed lines). The blue short-dashed line shows the resulting spectrum when the escape rate is $3 \times 10^{8} \mathrm{~g} \mathrm{~s}^{-1}$ and the ionizing EUV flux is 0.1 times the solar value. With these values, in particular the low EUV flux, the blue side of the line is absorbed by about $25 \%$, resulting in a decrease of about $13 \%$ in the total Lyman- $\alpha$ flux. The red long-dashed line shows the theoretical spectrum of the Lyman- $\alpha$ line during transit for an escape rate of $10^{10} \mathrm{~g} \mathrm{~s}^{-1}$ and an ionizing EUV flux 20 times the solar value. In this last case, the total Lyman- $\alpha$ flux decreases by about $5 \%$ when the exosphere passes in front of the star.

The profile of the Lyman- $\alpha$ stellar emission line seen from Earth is composed of the profile of the intrinsic stellar line combined with the absorption by the interstellar atomic hydrogen and deuterium. Interstellar hydrogen produced a narrow absorption at the line center. Deuterium absorption is weaker and blueshifted by $80 \mathrm{~km} \mathrm{~s}^{-1}$. The column density of atomic hydrogen toward HD 189733b is not known, but a simple relation between the stars' distance and the H I column density can be used. Using the results of Wood et al. (2005) and a distance of $19.3 \mathrm{pc}$ for HD $189733 \mathrm{~b}$, we infer an $\mathrm{H}$ I column of density, $N_{\mathrm{HI}}$, of about $10^{18.3} \mathrm{~cm}^{-2}$. Our conclusions are not sensitive to this assumption because the column density for a star at $\sim 20$ parsecs is typically in the flat part of the curve of growth. The core of the stellar line is fully absorbed by the cold interstellar medium. The width of the stellar Lyman- $\alpha$ emission line is obtained using the empirical relationship between the star absolute magnitude and the chromospheric Lyman- $\alpha$ line FWHM (Landsman \& Simon 1993). This relation can be written as $W_{\AA}=1.9 \times 10^{-M_{V} / 12.4}$, where the width $W_{\AA}$ is given in Angstroms. With an absolute $V$ magnitude of 6.1 , we infer a line width of about $0.61 \AA$, which is $F W M H=150 \mathrm{~km} \mathrm{~s}^{-1}$, or for a Gaussian $\sigma_{\mathrm{Ly} \alpha}=64 \mathrm{~km} \mathrm{~s}^{-1}$. We modeled the double peak chromospheric emission line with two Gaussians of width $\sigma_{\text {Ly } \alpha} / 2$ and separated by $\sigma_{\text {Ly } \alpha} / 2$. Combined with the interstellar absorption, we obtained the profile of the HD 189733 Lyman- $\alpha$ shown in Fig. 10.

\section{2. $H D 189733 b$ is evaporating}

We measure a $5 \%$ absorption depth over the broad Lyman- $\alpha$ line with a width of $\sim 150 \mathrm{~km} \mathrm{~s}^{-1}$, and this can be interpreted by two extreme scenarios. In the first scenario, the hydrogen cloud occults the full line width. In this case, the 5\% absorption corresponds to a cloud of about 1.65 Jupiter radii in size with gas velocities reaching over $150 \mathrm{~km} \mathrm{~s}^{-1}$. However, at 1.65 Jupiter radii the escape velocity from HD $189733 \mathrm{~b}$ is only $49 \mathrm{~km} \mathrm{~s}^{-1}$ (the escape velocity is $59 \mathrm{~km} \mathrm{~s}^{-1}$ at one planetary radius). In this first scenario, the hydrogen gas detected in the Lyman- $\alpha$ transit light curve exceeds the escape velocity, and is therefore not gravitationally bound to the planet, so the detected gas must be escaping.

In a second scenario, the velocity of the occulting gas does not exceed the escape velocity, and so the absorption only occurs in the core of the Lyman- $\alpha$ line. Because only $1 / 6$ of the observed Lyman- $\alpha$ flux comes from the core of the line within the $49 \mathrm{~km} \mathrm{~s}^{-1}$ radial velocity limit, in this scenario the absorption of the core must be at least $30 \%$ to produce a final absorption of $5 \%$ in the full unresolved line. A $30 \%$ absorption corresponds to a cloud of about 4 Jupiter radii in size, which significantly exceeds the size of the Roche lobe of the planet (3.3 Jupiter radii). In this second scenario, the gas detected in the Lyman- $\alpha$ transit light curve is beyond the Roche lobe, and is therefore not gravitationally bound to the planet, so the detected gas must be escaping.

An intermediate case between these two extreme scenarios can be considered. Even if the Roche lobe was full of gas but not beyond its borders, and the gas velocity was up to the escape velocity but not above it, the absorption depth would only be $3.3 \%$. Therefore, the measured 5\% absorption depth shows that some gas must be either beyond the Roche lobe or at a velocity above the escape velocity. The reality is most likely in between the two extreme scenarios with both a fraction of gas outside the Roche lobe and a fraction of gas at high velocity. The present observations of a 5\% absorption of the broad Lyman- $\alpha$ emission line demonstrates that HD 189733 b must be evaporating.

\subsection{Numerical simulation}

To interpret the HI light curve in more detail, we modeled the atmospheric gas escaping HD 189733b with a numerical simulation including the dynamics of the hydrogen atoms. In this $N$-body simulation, hydrogen atoms are released from HD 189733b's upper atmosphere with a random initial velocity corresponding to a $10000 \mathrm{~K}$ exobase. This temperature corresponds to the temperature expected in the upper atmosphere (e.g., Lecavelier des Etangs et al. 2004; Stone \& Proga et al. 2009) and has been actually measured for HD 209458b (Ballester et al. 2007). In any case, our results do not depend on this assumption because atoms are rapidly accelerated by the radiation pressure to velocities several times higher than their initial velocities. In this model, the gravity of both the star and the planet are taken into account. The radiation pressure from the stellar Lyman- $\alpha$ emission line is calculated as a function of 
the radial velocity of the atoms. The self-extinction within the cloud is also taken into account.

The hydrogen atoms are supposed to be ionized by the stellar extreme-UV (EUV). In the simulation, their lifetime is calculated as a function of the EUV flux, given as an input parameter to the model and expressed in solar units. The second input parameter is the atomic hydrogen escape rate, expressed in grams per second.

The dynamical model provides a steady state distribution of positions and velocities of escaping hydrogen atoms in the cloud surrounding HD 189733b. From this information we calculated the corresponding absorption over the stellar line and the corresponding transit light curve of the total Lyman- $\alpha$ flux to be directly compared with the observations.

\subsection{Results}

We calculated a grid of theoretical light curves as a function of the two input parameters, namely the escape rate and the EUV ionizing flux, and compared them to the measured Lyman- $\alpha$ flux. The EUV flux from HD 189733 has never been measured and is unknown. However, for a K-type star, it can be estimated to be in the range 1 to 100 times the solar EUV flux (e.g., Schmitt \& Liefke 2004).

Using the theoretical light curves, we fitted the observational data with the escape rate and EUV flux as free parameters. For an escape rate below $10^{7} \mathrm{~g} \mathrm{~s}^{-1}$, the occultation by the HI cloud is negligible and the resulting Lyman- $\alpha$ light curve is similar to the planet occultation light curve with a transit depth of $\sim 2.4 \%$ (Fig. 11). For a large escape rate $\left(\mathrm{d} M / \mathrm{d} t \gtrsim 10^{9} \mathrm{~g} \mathrm{~s}^{-1}\right)$ and low EUV flux ( $\$ 3$ times solar), the planet has a long trailing $\mathrm{H}$ I cloud with a cometary shape, and the occultation is deeper and lasts longer than the optical occultation by the planetary disk alone. With these last conditions, the Lyman- $\alpha$ transit depth exceeds $10 \%$ (Fig. 12). Both the transit depth and duration constrains the two parameters. Therefore, the fit to the Lyman- $\alpha$ light curve can be used to constrain both the escape rate and the ionizing flux (Fig. 13). We find that the data require an escape rate between $10^{9}$ and $10^{11} \mathrm{~g} \mathrm{~s}^{-1}$ and an EUV flux between 10 and 40 times the solar ionizing flux $(1-\sigma)$, with larger escape rates corresponding to larger EUV flux (Fig. 12). The best fit is obtained for $\mathrm{d} M / \mathrm{d} t=10^{10} \mathrm{~g} \mathrm{~s}^{-1}$ and EUV flux $F_{\text {EUV }}=20 F_{\odot}$. With these parameter values, the fit to the data with the evaporating planet model yields a $\chi^{2}$ of 91 for 80 degrees of freedom (Fig. 11). This fit is to be compared to the fit with a model of a single opticallythick disk for which we have a $\chi^{2}$ of 97.9 for 81 degrees of freedom (Table 3). Although the number of degrees of freedom is decreased by one in the evaporating planet model (because there are two free parameters, whereas only one parameter is needed to describe the size of the optically-thick disk), this fit is significantly better showing that the evaporating planet provides a better scenario to explain the observational data.

\section{Discussion}

The model used to interpret the H I transit light curve assumes a given Lyman- $\alpha$ line width of $0.61 \AA$ (Sect 5.1). This value is rather uncertain and might be underestimated (Landsman \& Simon 1993), and this is strengthened by the particularly high chromospheric activity of this star (Henry \& Winn 2008; Boisse et al. 2009). We tested this assumption by fitting the data as in Sects. 5.3 and 5.4, but using an FWMH of $1.0 \AA$ for the simulated HD 189733 Lyman- $\alpha$ stellar line. We found that, to fit the

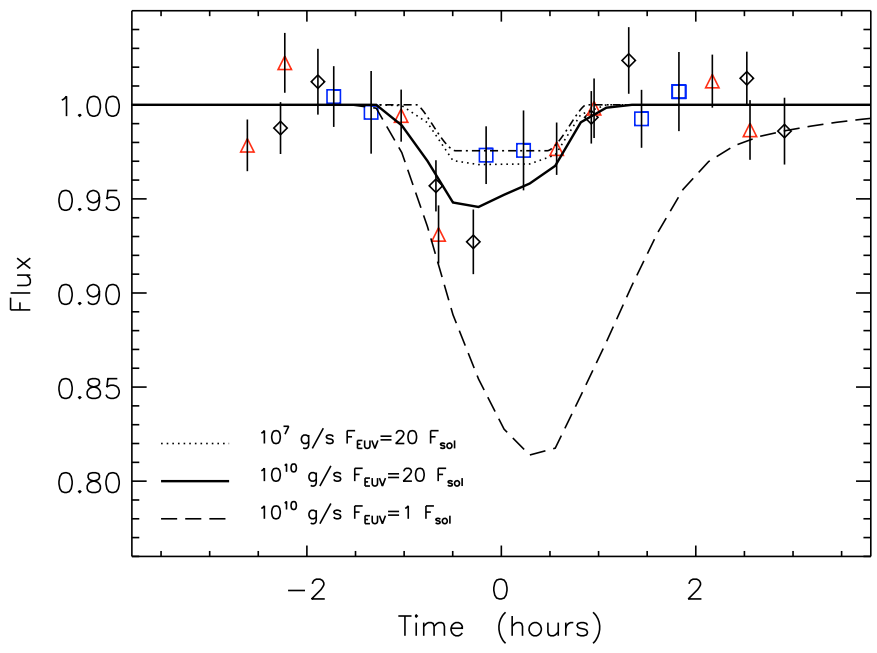

Fig. 11. Plot of the theoretical light curve of the total Lyman- $\alpha$ flux for various escape rates and ionizing EUV fluxes. The light curve obtained with an escape rate of $10^{10} \mathrm{~g} \mathrm{~s}^{-1}$ and an ionizing EUV flux of 20 times the solar value (thick line) best fits the data (symbols as in Fig. 6) with a resulting $\chi^{2}$ of 91 for 80 degrees of freedom. The dot-dashed line shows the light curve for a planet radius of 0.1564 times the stellar radius and no additional absorption by the planetary atmosphere.

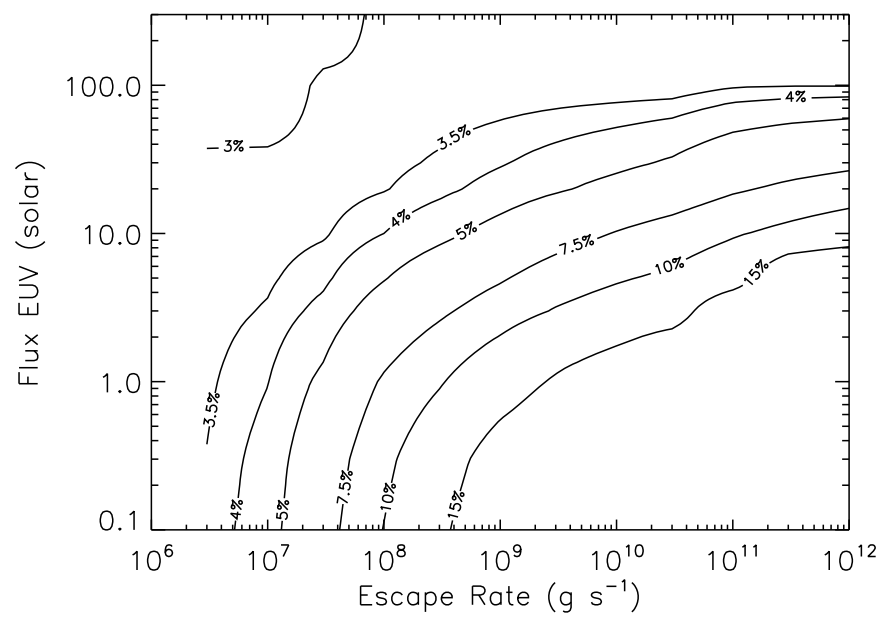

Fig. 12. Plot of the absorption depth on the theoretical light curve of the stellar H I Lyman- $\alpha$ line as a function of the escape rate and EUV ionizing flux.

observed light curve, a broader line requires a lower EUV ionizing flux, because hydrogen atoms need to reach higher velocities to occult the broader wings of the line before being ionized. We found that an EUV ionizing flux between 2 and 10 times the solar value and similar escape rates as in Sect. 5 provide a good fit to the data. Taking the uncertainty on the Lyman- $\alpha$ line width into account, we ended up with an EUV ionizing flux between 2 and 40 times the solar value $(1-\sigma)$. This test shows that the estimate of the escape rate is not sensitive to the assumption on the width of the stellar emission line.

We constrained the escape rate of atomic hydrogen to be about $10^{10} \mathrm{~g} \mathrm{~s}^{-1}$, which is similar to the escape rate previously estimated in the case of HD 209458b. The similarity between the two escape rates can be explained by comparing the energy input by the stars and the physical properties of the planets. In effect, theoretical models of the evaporation of hot Jupiters agree to conclude that the escape rate can be estimated by assuming that the EUV and X-ray energy input from the star is used by the 


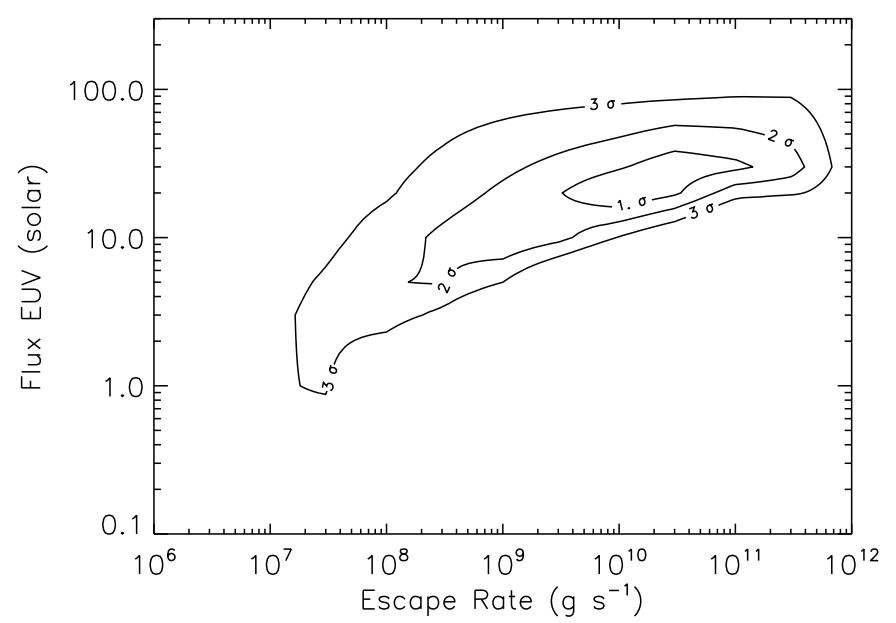

Fig. 13. Plot of error bars for the estimated escape rate and EUV ionizing flux. The best fit is obtained for an escape rate of $10^{10} \mathrm{~g} \mathrm{~s}^{-1}$ and an ionizing EUV flux of 20 times solar. To fit the data at $2 \sigma$ confidence level, the escape is estimated to be between $10^{8}$ and $3 \times 10^{11} \mathrm{~g} \mathrm{~s}^{-1}$ and the EUV flux between 5 and 60 times the solar value. The escape rate needed to fit the data is correlated with the ionizing flux, with large escape rate for a larger ionizing flux.

gas to escape the gravitation of the planet (Lecavelier des Etangs 2007). The potential well of HD 189733 b is about twice deeper than that of HD 209458b (the planet mass is 1.7 larger, and the radius is $12 \%$ smaller). However, this well is largely compensated for by the orbital distance of HD 189733b which is 1.5 times closer to its star than HD 209458b and the stellar type of HD 189733 which as a K-type star is expected to provide a higher EUV and X-ray input energy. Within the uncertainties, these orders of magnitude show that the similarity between the observed evaporations of HD 189733b and HD 209458b results from their similar physical properties. In both cases, the observed escape rate is modest, and even if the escape of ionized hydrogen is not detectable in Lyman- $\alpha$, evaporation is unlikely to change the nature of these two planets: $10^{10} \mathrm{~g} \mathrm{~s}^{-1}$ corresponds to the escape of about $0.2 \%$ of the planets' mass in 10 billion years.

However, the net effect of variations in EUV flux on the observable is not obvious. In effect, although an increased stellar EUV flux deposits more energy onto the planetary upper atmosphere, and thereby increasing the heating and consequently the atmospheric escape (e.g., Lecavelier des Etangs et al. 2004), it is also more efficient at ionizing the escaping $\mathrm{H}$ I atoms, hence reducing the size of the observable $\mathrm{H}$ I cloud measured during the transit. This also contributes to explaining why we find comparable absorption depths on HD 189733b and HD 209458b, although their host stars are of different stellar types. Here it must be recalled that the transit depth is measured on the Lyman- $\alpha$ line of atomic hydrogen and is interpreted in terms of escape rate through a model in which the hydrogen ions are produced by ionization by EUV stellar photons; therefore, the quoted escape rates do not include escape of hydrogen ionized by other mechanisms lower in the atmosphere.

We did not detect a transit signature of species other than hydrogen, because of the photon noise limit on individual lines. The transit signature can be seen by adding all photons redward of the Lyman- $\alpha$ including all lines and the stellar continuum; in this case the transit depth is consistent with the absorption by the planetary disk alone. With this limitation, it is not possible to conclude anything about the escape mechanism, contrary to the case of HD 2908458 b where detection of oxygen and carbon revealed the hydrodynamical escape ("blow-off") of the atmosphere (Vidal-Madjar et al. 2004).

\section{Conclusion}

In summary, we have presented the $3.5 \sigma$ detection of an excess absorption in the Lyman- $\alpha$ transit light curve. This is interpreted in terms of the evaporation in the upper atmosphere of HD 189733b. Any detection at this level of confidence would call for an observational confirmation, which will be possible with the repaired STIS spectrograph. If confirmed, HD 189733b would be the second extrasolar planet for which evaporation has been detected.

In the present observations, the Lyman- $\alpha$ line is not resolved, removing any direct information on the radial velocity of the absorbing gas. This limitation is due to the limited spectroscopic capabilities of the ACS instrument in the far-ultraviolet, and the ACS was used because of the STIS failure. Fortunately, a significant improvement is expected after the refurbishment of the Hubble Space Telescope performed last May 2009. With the new Cosmic Origins Spectrograph (COS) and the repaired STIS, new observations should allow us to address the issue of the escape mechanism and to probe the dynamics of escaping atoms through an improved sensitivity and sampling of the absorption along the Lyman- $\alpha$ line profile.

Finally, the results from each of the three observed transits of HD 189733 b are not exactly the same (Table 3). Since the observations were obtained at different epochs (the last transit was observed ten months later than the first two ones), this raises the question of possible time variations in the transit depth. From the present data, the detection of variations is barely significant $(\sim 2.5 \sigma)$, and it may not be possible to draw definite conclusions on this question. Nonetheless, variations would not be surprising, at least because of the expected variations in the EUV ionizing flux which, for a given escape rate, significantly affects the observed transit depth (Fig. 12). Interpretation of observations made at a single epoch is always tricky, and any estimate or nondetection at a given time should never be considered definitive.

Acknowledgements. We warmly thank F. Pont and F. Bouchy for useful comments and discussions, and A. Baillard and M. Dennefeld for their OHP observations of the HD 189733 field for preparing our HST observations.

Based on observations made with the NASA/ESA Hubble Space Telescope, obtained at the Space Telescope Science Institute, which is operated by the Association of Universities for Research in Astronomy, Inc., under NASA contract NAS 5-26555. These observations are associated with program GO10869. D.E. acknowledges financial support from the French Agence Nationale pour la Recherche (ANR; project NT05-4_44463) and from the Centre National d'Études Spatiales (CNES). G.E.B. acknowledges financial support by this program through STScI grant HST-GO-10869.01-A to the University of Arizona.

\section{References}

Bakos, G. Á., Knutson, H., Pont, F., et al. 2006, ApJ, 650, 1160 Ballester, G. E., Sing, D. K., \& Herbert, F. 2007, Nature, 445, 511 Baraffe, I., Selsis, F., Chabrier, G., et al. 2004, A\&A, 419, L13 Baraffe, I., Chabrier, G., Barman, T. S., et al. 2005, A\&A, 436, L47 Baraffe, I., Alibert, Y., Chabrier, G., \& Benz, W. 2006, A\&A, 450, 1221 Ben-Jaffel, L. 2007, ApJ, 671, L61

Ben-Jaffel, L., \& Sona Hosseini, S. 2010, ApJ, 709, 1284

Bentley, S. J., Hellier, C., Maxted, P. F. L., et al. 2009, A\&A, 505, 901 Boisse, I., Moutou, C., Vidal-Madjar, A., et al. 2009, A\&A, 495, 959 Bouchy, F., Udry, S., Mayor, M., et al. 2005, A\&A, 444, L15 Brekke, P., Rottman, G. J., Fontenla, J., \& Judge, P. G. 1996, ApJ, 468, 418 Burrows, A., \& Lunine, J. 1995, Nature, 378, 333

Charbonneau, D., Knutson, H. A., Barman, T., et al. 2008, ApJ, 686, 1341 Désert, J.-M., Lecavelier des Etangs, A., Hébrard, G., et al. 2009, ApJ, 699, 478 
Ehrenreich, D. 2010, in Physics and Astrophysics of Planetary Systems, ed. T. Montmerle, D. Ehrenreich, \& A.-M. Lagrange (EDP Sciences), EAS Publ. Ser., 41,429

Ehrenreich, D., Lecavelier des Etangs, A., Hébrard, G., et al. 2008, A\&A, 483, 933

García Muñoz, A. 2007, Planet. Space Sci., 55, 1426

Gaudi, B. S., Seager, S., \& Mallen-Ornelas, G. 2005, ApJ, 623, 472

Grillmair, C. J., Burrows, A., Charbonneau, D., et al. 2008, Nature, 456, 767

Guillot, T., Burrows, A., Hubbard, W. B., Lunine, J. I., \& Saumon, D. 1996, ApJ, 459, L35

Hébrard, G., \& Lecavelier des Etangs, A. 2006, A\&A, 445, 341

Hébrard, G., Lemoine, M., Vidal-Madjar, A., et al. 2002, ApJS, 140, 103

Henry, G. W., \& Winn, J. N. 2008, AJ, 135, 68

Holmström, M., Ekenbäck, A., Selsis, F., et al. 2008, Nature, 451, 970

Jaritz, G. F., Endler, S., Langmayr, D., et al. 2005, A\&A, 439, 771

Knutson, H. A., Charbonneau, D., Cowan, N. B., et al. 2009, ApJ, 690, 822

Lammer, H., Selsis, F., Ribas, I., et al. 2003, ApJ, 598, L121

Landsman, W., \& Simon, T. 1993, ApJ, 408, 305

Lecavelier des Etangs, A. 2007, A\&A, 461, 1185

Lecavelier des Etangs, A., Vidal-Madjar, A., Hébrard, G., \& McConnell, J. 2004, A\&A, 418, L1

Lecavelier des Etangs, A., Vidal-Madjar, A., \& Desert, J.-M. 2008a, Nature, 456, E1

Lecavelier des Etangs, A., Pont, F., Vidal-Madjar, A., \& Sing, D. 2008b, A\&A, 481, L83

Lecavelier des Etangs, A., Sirothia, S. K., \& Gopal-Krishna, Z. P. 2009, A\&A, 500, L51
Léger, A., Rouan, D., Schneider, J., et al. 2009, A\&A, 506, 287 Mayor, M., \& Queloz, D. 1995, Nature, 378, 355

Moutou, C., Donati, J.-F., Savalle, R., et al. 2007, A\&A, 473, 651

Murray-Clay, R. A., Chiang, E. I., \& Murray, N. 2009, ApJ, 693, 23

Pont, F., Gilliland, R. L., Moutou, C., et al. 2007, A\&A, 476, 1347

Pont, F., Knutson, H., Gilliland, R. L., Moutou, C., \& Charbonneau, D. 2008, MNRAS, 385, 109

Redfield, S., Endl, M., Cochran, W. D., \& Koesterke, L. 2008, ApJ, 673, L87

Schmitt, J. H. M. M., \& Liefke, C. 2004, A\&A, 417, 651

Sing, D. K., Desert, J.-M., Lecavelier des Etangs, A., et al. 2009, A\&A, 505, 891 Stone, J. M., \& Proga, D. 2009, ApJ, 694, 205

Swain, M. R., Vasisht G., \& Tinetti, G. 2008, Nature, 452, 329

Tian, F., Toon, O. B., Pavlov, A. A., \& De Sterck, H. 2005, ApJ, 621, 1049

Vidal-Madjar, A., \& Lecavelier des Etangs, A. 2004, Extrasolar Planets: Today and Tomorrow, ASP Conf. Ser., 321152

Vidal-Madjar, A., Lecavelier des Etangs, A., Désert, J.-M., et al. 2003, Nature, 422,143

Vidal-Madjar, A., Désert, J.-M., Lecavelier des Etangs, A., et al. 2004, ApJ, 604, L69

Vidal-Madjar, A., Lecavelier des Etangs, A., Désert, J.-M., et al. 2008, ApJ, 676, 57

Wood, B. E., Redfield, S., Linsky, J. L., Müller, H.-R., \& Zank, G. P. 2005, ApJS, 159,118

Winn, J. N., Holman, M. J., Henry, G. W., et al. 2007, AJ, 133, 1828

Yelle, R. V. 2004, Icarus, 170, 167

Yelle, R. V. 2006, Icarus, 183, 508 